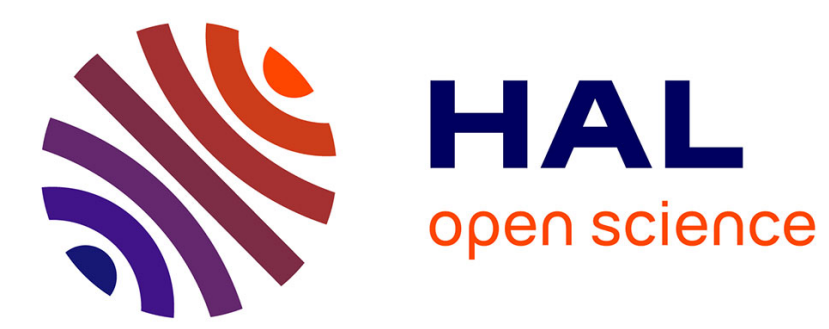

\title{
Dynamical analysis of magnetic field effects on a cholesteric cano wedge
}

G. Malet, J. Marignan, O. Parodi

\section{To cite this version:}

G. Malet, J. Marignan, O. Parodi. Dynamical analysis of magnetic field effects on a cholesteric cano wedge. Journal de Physique Lettres, 1975, 36 (12), pp.317-320. 10.1051/jphyslet:019750036012031700 . jpa-00231218

\section{HAL Id: jpa-00231218 https://hal.science/jpa-00231218}

Submitted on 1 Jan 1975

HAL is a multi-disciplinary open access archive for the deposit and dissemination of scientific research documents, whether they are published or not. The documents may come from teaching and research institutions in France or abroad, or from public or private research centers.
L'archive ouverte pluridisciplinaire HAL, est destinée au dépôt et à la diffusion de documents scientifiques de niveau recherche, publiés ou non, émanant des établissements d'enseignement et de recherche français ou étrangers, des laboratoires publics ou privés. 


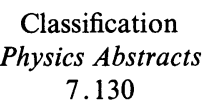

\title{
DYNAMICAL ANALYSIS OF MAGNETIC FIELD EFFECTS ON A CHOLESTERIC CANO WEDGE
}

\author{
G. MALET, J. MARIGNAN and O. PARODI
}

Groupe de Dynamique des Phases Condensées $\left({ }^{*}\right)$, Laboratoire de Minéralogie et de Cristallographie, Université des Sciences et Techniques du Languedoc, Place Eugène-Bataillon, 34060 Montpellier, France

(Reçu le 16 juillet 1975, révisé le 11 septembre 1975, accepté le 29 septembre 1975)

\begin{abstract}
Résumé. - Nous présentons brièvement quelques résultats expérimentaux et théoriques sur l'étude dynamique du déplacement de la première ligne de Grandjean-Cano, dans un cholestérique soumis à des champs magnétiques relativement faibles. Ce déplacement est caractérisé par une grande valeur du temps de relaxation due à une forte viscosité apparente. Le bon accord entre l'expérience et la théorie permet d'atteindre le rayon du cœur de la ligne.
\end{abstract}

\begin{abstract}
We present briefly the main experimental and theoretical results concerning the dynamics of the first Grandjean-Cano disclination line in the presence of weak magnetic fields. The motion of the line is characterized by a long relaxation time connected with the large value of the apparent viscosity. The good agreement between experiments and theory should provide the core radius of the line.
\end{abstract}

1. Introduction. - It has been known for a long time that a cholesteric sample placed in a variable thickness cell (Cano wedge), shows a set of disclination lines known as Grandjean-Cano lines; their positions are related to the cholesteric pitch $P$, these lines appearing at places where the sample thickness $d=(2 n+1) P / 4[1]$.

If a magnetic field normal to the helical axis is applied to the cholesteric sample with positive magnetic anisotropy $\left(\chi_{\mathrm{a}}>0\right)$, the pitch increases and, as a consequence, the Grandjean-Cano lines move to new equilibrium positions. A static theory of this effect has been developed by de Gennes [2] and Meyer [3], and successful experimental verifications have been performed by the Orsay [4] and Harvard [5] groups.

The dynamics of this effect has not yet been analysed for cholesterics, though a similar problem has been independently studied by Geurst et al. in twisted nematics [6].

The purpose of this letter is to present briefly the main experimental and theoretical results concerning the dynamics of the first single line for weak applied magnetic fields. A more detailed paper will be publishshed later.

In part 2 a description of the experimental apparatus is given.

In part 3 the basic assumption and the main results of theoretical approach are presented. This approach is limited to the analysis of the dynamics of the first

${ }^{*}$ ) Laboratoire associé au C.N.R.S. single line under weak magnetic fields : $H<H_{\mathrm{c}}$ where $H_{c}$ is the critical field for the cholestericnematic transition. The experimental results for this line are given in part 4 and compared with the theoretical predictions.

2. Experimental apparatus. - In order to obtain cholesterics with positive $\chi_{a}$, the simplest procedure is to dissolve chiral molecules in a conventional nematic; in order to have reasonable critical field values, one has to work with large pitch mixtures, i.e. for small concentrations. One advantage of this procedure is that experimental studies in magnetic fields are much easier when the cholesteric state occurs at room temperature.

We have therefore used cholesterol cinnamate diluted in an equimolar mixture of 4-methoxy-4'penthyl-tolane and 4-propoxy-4'-heptyl-tolane [7]. The cholesteric concentration is $c=0.63 \%$.

The liquid crystal is introduced between a cylindrical lens of $8 \mathrm{~cm}$ radius and a flat plate (Cano wedge). The glasses are precoated with thin evaporated films of $\mathrm{SiO}$ under oblique incidence in order to obtain a strong anchoring with easy direction normal to the contact generator [8].

The sample is placed in a magnet and observed with a non magnetic microscope. The positions of Grandjean-Cano lines are located using a micrometer eyepiece; a photographic survey allows for measuring displacements of lines versus time. 
The magnetic field (variable from 0 to $10000 \mathrm{G}$ for an air gap of $5 \mathrm{~cm}$ ) is applied uniformly along the anchoring direction, i.e. normal to the helical axis.

Experimental studies have been carried out on four single lines and three double lines, under different values of the applied magnetic field $\left(H \gtrless H_{\mathrm{c}}\right)$. A set of lines is shown on figure 1 . The behaviour of the double lines is not very different. It will not be discussed in this letter.

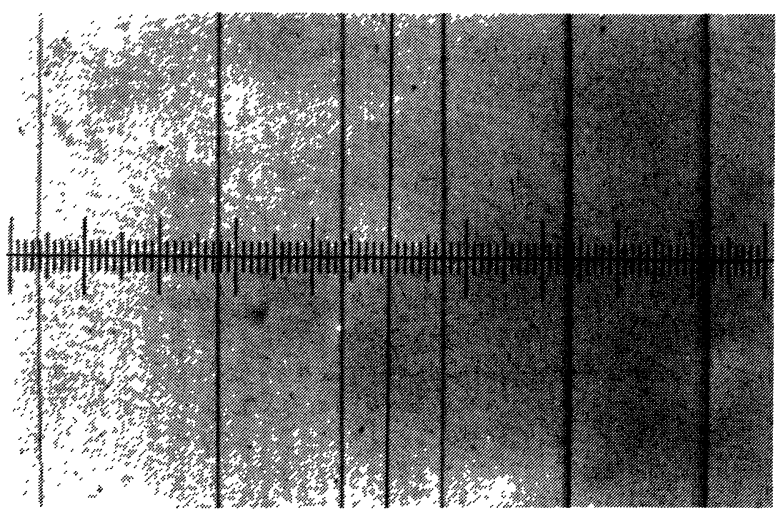

Fig. 1. - Four single Grandjean-Cano lines and two double lines in a cholesteric-Cano wedge : $c=0.63 \%, P_{0}=29.7 \mu \mathrm{m}, H=0$.

The contact generator is on the left of the photograph.

3. Theoretical analysis. - It is worth pointing out that our experimental results lead to very long relaxation times for the line motion. As a consequence, the relaxation process can be separated in two independents parts. The first one, leads to a reorientation of the director under the magnetic field in a very short time $\left(\tau \simeq 10^{-2} \mathrm{~s}\right)$, without any motion of the lines. The second relaxation process, characterized by a much larger relaxation time $\left(\tau \simeq 10^{2} \mathrm{~s}\right)$, drives the lines toward their equilibrium position.

3.1 FAST REORIENTATION PROCESS. - Consider an incompressible cholesteric slab with the helical axis along the $Z$ axis (Fig. 2). The strong anchoring direction and the applied magnetic field are both taken along the $Z$ axis. The first disclination line is taken at the origin. Then, for $x<0$, the sample has a nematic configuration, and for $x>0$, the period of the cholesteric is $d$, where $d$ is the local sample

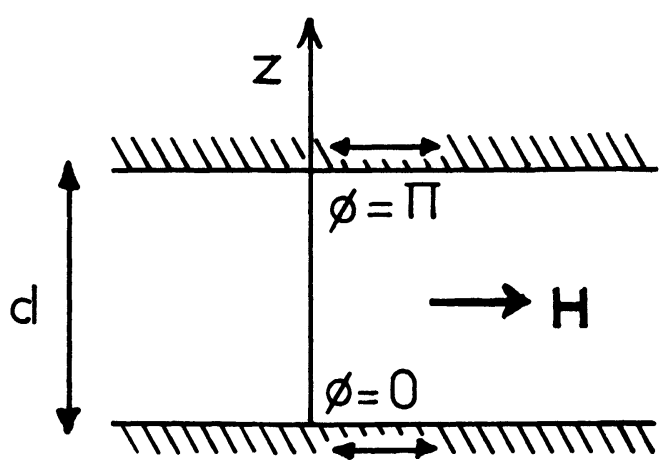

FIG. 2. - Cholesteric slab under a magnetic field parallel to the strong anchoring direction and normal to the helical axis. thickness (the local pitch being $2 d$ ). The only deformation involved is twist, and the director $\mathbf{n}$ has components :

$$
\begin{aligned}
& n_{x}=\cos \varphi \\
& n_{y}=\sin \varphi \\
& n_{z}=0 .
\end{aligned}
$$

It can be easily shown that the bend and splay deformations resulting from the thickness variation can be neglected, so that we can suppose for the following analysis that $d$ is constant and take the $z$ origin at the middle of the slab (Fig. 2).

The $(\mathbf{O X}, \mathbf{n})$ angle is therefore a function of $z$ and $t$ and the boundary conditions are $\varphi=0$ for $z=-d / 2$ and $\varphi=\pi$ for $z=d / 2$.

Using standard notations, the Frank free energy per unit surface is given by

$F_{\mathrm{c}}=\int_{-d / 2}^{d / 2}\left[\frac{K_{2}}{2}\left(\frac{\partial \varphi}{\partial z}-q_{0}\right)^{2}-\frac{\chi_{\mathrm{a}} H^{2}}{2} \cos ^{2} \varphi\right] \mathrm{d} z$.

The relaxation process involves only a change in twist. Then it is well known from the Leslie equations, that such a reorientation is not coupled to any hydrodynamic backflow. The entropy source $T \dot{S}$ per unit area is therefore :

$$
T \dot{S}=\int_{-d / 2}^{d / 2} \gamma_{1}\left(\frac{\partial \varphi}{\partial t}\right)^{2} \mathrm{~d} z
$$

For an isothermal process, the dissipation $T \dot{S}$ is equal to the decrease in stored free energy

$$
T \dot{S}=\frac{-\mathrm{d} F_{\mathrm{c}}}{\mathrm{d} t} .
$$

Eq. (4) gives the dynamical equation for $\varphi(z, t)$ and its solution under weak magnetic fields $\left(H<H_{\mathrm{c}}\right)$ can be expressed in the following terms :

$$
\varphi(z, t)=\frac{\pi}{2}+\frac{\pi z}{d}+a(t) \sin \frac{2 \pi z}{d}
$$

where

$$
a(t)=\frac{d^{2}}{8 \xi^{2} \pi^{2}}\left(1-\mathrm{e}^{-t / \tau}\right) .
$$

Here $\xi=\left(K_{2} / \chi_{\mathrm{a}}\right)^{1 / 2} / H$ is the magnetic coherence length and the caracteristic relaxation time is given by

$$
\tau=\frac{\gamma_{1} d^{2}}{4 \pi^{2} K_{2}} .
$$

Taking $d=10 \mu \mathrm{m}, K_{2} \simeq 5 \times 10^{-7} \mathrm{dyn}, \gamma_{1}=10^{-1} \mathrm{P}$, one gets $\tau \simeq 2 \times 10^{-2} \mathrm{~s}$, in good agreement with our a priori assumption.

3.2 DyNamics OF THE FIRST GRANDJEAN-CANO LINE. - Consider now a Cano wedge (Fig. 3). Our frame of reference has already been defined. The $y$ axis is taken along the disclination line at time $t=0$, when the magnetic field is increased from $H_{0}$ to $H$. 
The line now moves and has abscissa $x(t)$. The corresponding sample thickness is

$$
\mathrm{d}(t)=\frac{\left(x(t)+x_{0}\right)^{2}}{2 R}
$$

where $-x_{0}$ is abscissa of the contact generator and $R$ the radius of the lens.

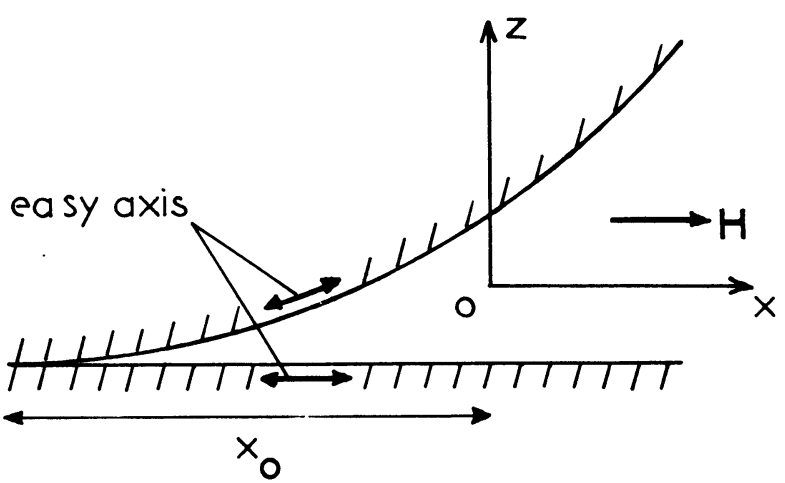

Fig. 3. - Cholesteric sample in cylindrical geometry (Cano wedge) The equilibrium position of the first single Grandjean-Cano line, under initial magnetic field $H_{0}$, is assumed to be along the $y$-axis.

The sample can be divided in three parts.

For $x<x(t)-d(t)$, it has a nematic configuration. For $x>x(t)+d(t)$, it has a distorted cholesteric configuration. The disclination introduces a strong distortion in the range $x(t)-d, x(t)+d$. The free energy of this strong distorted domain can be considered as time independent. Then the free-energy variation per unit length along the $y$-axis and per unit time is :

$$
\frac{\mathrm{d} F}{\mathrm{~d} t}=\left(F_{\mathrm{n}}-F_{\mathrm{c}}\right) \frac{\mathrm{d} x}{\mathrm{~d} t}
$$

where $F_{\mathrm{c}}$ is the cholesteric free-energy per unit area, as deduced from eq. (5) and (6) :

$$
F_{\mathrm{c}}=\frac{K_{2} d}{2}\left(\frac{\pi}{d}-q_{0}\right)^{2}-\frac{\chi_{\mathrm{a}} H^{2} d}{4}
$$

and $F_{\mathrm{n}}$ is the free energy per unit area of the nematic domain

$$
F_{\mathrm{n}}=\frac{K_{2}}{2} q_{0}^{2} d-\frac{\chi_{\mathrm{a}} H^{2} d}{2} .
$$

The dissipation $T \dot{S}$ results from the reorientation which essentially occurs in the strong distorted domain around the disclination line

$$
T \dot{S}=\left(\frac{\mathrm{d} x}{\mathrm{~d} t}\right)^{2} \int_{-\infty}^{+\infty} \mathrm{d} x \int_{-d / 2}^{d / 2} \gamma_{1}\left(\frac{\partial \varphi}{\partial x}\right)^{2} \mathrm{~d} z
$$

In eq. (12), $\partial \varphi / \partial x$ is given by de Gennes planar model around a disclination [9]. A complete calculation gives :

$$
\begin{aligned}
\varphi\left(x, z_{0}\right) & =\frac{\pi}{2} E(x)+\frac{\pi}{2 d} z+ \\
& +\frac{1}{2} \operatorname{Arctan}\left[\operatorname{coth}\left(\frac{K_{2}}{K_{1}}\right)^{1 / 2} \frac{\pi x}{d} \tan \frac{\pi z}{d}\right]
\end{aligned}
$$

and

$$
\frac{\partial \varphi}{\partial x}=-\left(\frac{K_{2}}{K_{1}}\right)^{1 / 2} \frac{\pi}{2 d} \frac{\sin \frac{2 \pi z}{d}}{\cosh \left(\frac{K_{2}}{K_{1}}\right)^{1 / 2} \frac{2 \pi x}{d}-\cos \frac{2 \pi z}{d}} .
$$

$E(x)$ is the step function $(E(x)=0$ for $x<0$; $E(x)=1$ for $x>0$ ).

It must be noted that $\partial \varphi / \partial x[10]$ has a notable variation only near the disclination, which justifies keeping $d$ constant (at fixed $t$ ) in eq. (12).

Then, using eq. (9) and (12) one obtains a differential equation for $x(t)$, with solution, for $H<H_{\mathrm{c}}$

$$
\begin{aligned}
t=\tau_{-} & \left(\tanh ^{-1} \frac{x+x_{0}}{l_{-}}-\tanh ^{-1} \frac{x_{0}}{l_{-}}\right)- \\
& \quad-\tau_{+}\left(\tanh ^{-1} \frac{x+x_{0}}{l_{+}}-\tanh ^{-1} \frac{x_{0}}{l_{+}}\right) .
\end{aligned}
$$

Here, $\tau_{-}$, and $\tau_{+}$are two relaxation times :

$$
\tau_{ \pm}=\frac{2 \gamma_{1} V \sqrt{1 \pm \alpha}}{\alpha \chi_{\mathrm{a}} H^{2} \xi \sqrt{\pi q_{0} / R}}
$$

where

$$
V=\frac{\pi}{4}\left(\frac{K_{2}}{K_{1}}\right)^{1 / 2}\left[\ln \frac{d}{4 \pi \varepsilon}\left(\frac{K_{1}}{K_{2}}\right)^{1 / 2}+\frac{1}{2}+\frac{2 G}{\pi}\right] .
$$

The logarithmic divergence appearing in $V$ is usual in disclinations. $\varepsilon$ is a cut-off radius, the core radius of the disclination.

$G \simeq .916 \ldots$ is Catalan's constant

$$
\begin{aligned}
\alpha & =\left(1-H^{2} / H_{l}^{2}\right)^{1 / 2}, \quad H_{l}=\frac{2 \sqrt{2}}{\pi} H_{\mathrm{c}} \\
l_{ \pm} & =2 \xi \sqrt{q_{0} \pi R(1 \pm \alpha)} .
\end{aligned}
$$

4. Results. - Rewriting eq. (14) with the following notations

$$
\xi_{l}=\frac{1}{H_{l}}\left(\frac{K_{2}}{\chi_{\mathrm{a}}}\right)^{1 / 2}, f_{ \pm}=\frac{1}{\alpha \sqrt{1 \mp \alpha}}
$$

we obtain

$$
\tau_{ \pm}=\tau_{0} f_{ \pm}\left(H / H_{l}\right)
$$


where

$$
\tau_{0}=\frac{\gamma_{\text {eff }}}{\chi_{\mathrm{a}} H_{l}^{2}} \quad \gamma_{\text {eff }}=\frac{2 V \gamma_{1}}{\xi_{l} \sqrt{\pi q_{0} / R}}
$$

$\tau_{0}$ is the field independant relaxation time and $\gamma_{\text {eff }}$ an effective viscosity.

The physical meaning of the two relaxation times $\tau_{+}$ and $\tau_{-}$is not obvious. However one can easily see that $\tau_{0}$ is a pure elastic relaxation time

$$
\tau_{0}=\frac{\gamma_{1}}{K_{2} q_{0}^{2}} V \sqrt{\frac{2 q_{0} R}{\pi}} .
$$

For weak magnetic fields, one has

$$
\begin{aligned}
& \tau_{-} \simeq \frac{\tau_{0}}{\sqrt{2}}\left(1+\frac{5 H^{2}}{8 H_{l}^{2}}\right) \\
& \tau_{+} \simeq \tau_{0} \sqrt{2} \frac{H_{l}}{H}\left(1+\frac{H^{2}}{2 H_{l}^{2}}\right) .
\end{aligned}
$$

Therefore $\tau_{-}$appears, for low fields, as a pure elastic relaxation time. On the other hand $\tau_{+} \sim H^{-1}$ characterizes a magnetic relaxation which disappears $\left(1 / \tau_{+}=0\right)$ at zero-field.

We can then plot the theoretical curves giving $t / \tau_{0}$ versus $x$, for different values of $H_{0}$ and $H$. By fitting these curves with the experimental ones, we can obtain the value of $\tau_{0}$ and deduce the relaxation times $\tau_{-}$and apparent viscosity $\gamma_{\text {eff }}$.

For our sample, the unperturbed pitch is $P_{0}=29.7 \mu \mathrm{m}$ and the observed critical field for the cholesteric nematic transition is $H_{\mathrm{c}} \simeq 7700 \mathrm{G}$. Using these values we find $\gamma_{\text {eff }} \simeq 550 \mathrm{P}$.

The variation of time $t$ with line displacement $x$ is shown in figure 4. The initial magnetic field is $H_{0}=0$ and the applied one is $H=2960 \mathrm{G}$. The full line is the theoretical one, the open circles correspond to our experimental data. We find a relaxation time $\tau_{-}=90 \mathrm{~s}$.

5. Conclusion. - This theoretical approach on the dynamics of the Grandjean-Cano lines was essentially based on separating the analysis in two parts : adjustment of the director under field without motion of lines and then displacement of the lines themselves. The experimental and theoretical results concerning the first single line under weak magnetic fields are in good agreement. We note the large value of the apparent viscosity $\gamma_{\text {eff }}$ which is quite typical of line relaxation process, and as a consequence, explains the high relaxation times.

We should point out that if $\gamma_{1}$ and $K_{2} / \chi_{\mathrm{a}}$ are known, one can derive from eq. (15) and (16) the core radius of the line.

A more detailed version of this paper will be published later.

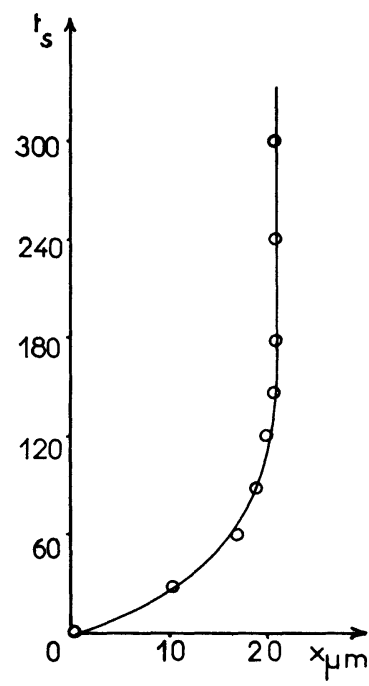

FIG. 4. - Time $t$ as a function of displacement $x$ of the first single line for a mixture MPT-PHT cholesterol cinnamate. $H=2960 \mathrm{G}$ $H_{0}=0$. The full line is theoretical. Open circles correspond to the experimental values.

\section{References}

[1] Cano, R., Bull. Soc. Fr. Miner. Cristallogr. 90 (1967) 333.

[2] De Gennes, P. G., Solid State Commun. 6 (1968) 163.

[3] Meyer, R. B., Appl. Phys. Lett. 12 (1968) 281.

[4] Durand, G., LéGer, L., Rondelez, F. and Veyssié, M., Phys. Rev. Lett. 22 (1969) 227.

[5] MeYer, R. B., Appl. Phys. Lett. 14 (1969) 208.

[6] Gerritsma, C. J., Geurst, T. A. and SpruiJt, A. N. J., Phys. Lett. 43A (1973) 356.

Geurst, T. A., Spruijt, A. N. J. and Gerritsma, C. J., $J$. Physique (to be published).
[7] Malthete, J., LeclercQ, M., Gabard, J., Billard, J. and JACQUES, J., C. R. Hebd. Séan. Acad. Sci. C 273 (1971) 265.

[8] Guyon, E., Pieranski, P. and Boix, M., Lett. App. Eng. Sci. 1 (1973) 19.

[9] Bouligand, Y., J. Physique 35 (1974) 959.

De Gennes, P. G., C. R. Hebd. Séan. Acad. Sci. B 266 (1968) 571.

[10] Malet, G., Marignan, J. and Parodi, O., J. Physique (to be published). 